\title{
The features of three- and four-tier internal dumps capacity calculation with the additional capacity preparation in the dump tiers
}

\author{
Tatiana Gvozdkova ${ }^{1, *}$, Eugene Kuznetsov ${ }^{1}$, Anna Rudakova $^{1}$, Sergei Markov ${ }^{2}$ \\ ${ }^{1}$ T.F. Gorbachev Kuzbass State Technical University, Mezhdurechensk branch, Mezhdurechensk, \\ 652881, Stroitelei Avenue, 36, Russia \\ ${ }^{2}$ T.F. Gorbachev Kuzbass State Technical University, Kemerovo, 650000, 28 Vesennyaya st., Russia
}

\begin{abstract}
The paper deals with the task of increasing the volume of rock placed in the internal dump. For that it is necessary first to analyze the existing technology of internal dumping, and then to determine the height of rock tiers being cut in the transport area. Analyzing the mining on the southern pits of Kuzbass it was found that now mostly three-tier dumps are piled. But for placing the extra volume of rock in the internal dump it will be required to pile dumps with more than three tiers.
\end{abstract}

\section{Introduction}

Transportless technology is now widely used on Kuzbass pits and in other coal producing regions. Its main advantage is the reduction of land capacity due to the possibility of placing internal dump in the worked out pit area, as well as lower cost of one cubic meter of overburden in comparison with truck-and-shovel technology [1]. As a result of low-dipping strata $\left(6-14^{\circ}\right)$ three-tier dumps are usually piled. In case of coincidence of two mutually influencing factors - the dip angle and the dragline beam length - there is a need for dumping piles with a large number of tiers [2]. There is an experience of the four-tier dumps, and it showed a certain complication of draglines' work on the dump and an increase in total coefficient of re-excavation by creating additional volumes of the secondary rehandling.

\section{Material and Method}

When piling five-tier dumps it is possible to expect a substantial increase in total coefficient of re-excavation and complication of draglines' work on the dump [3].

The height of cutting seam is the difference between the capacity of four-tier and threetier dumps. Dumps' capacity is represented as a height of the rock seam in an array equivalent to this capacity [4].

The height of cutting seam $H_{c u t}$ is, m:

\footnotetext{
*Corresponding author: kuzstu@kuzstu.su
} 


$$
H_{c u t}=\frac{E_{o .4}-E_{o .3}}{A \cdot K_{p}},
$$

where: $E_{0.4}$ - the four-tier dump capacity at 1 meter of mining front, $\mathrm{m}^{3} ; E_{0.3}$ - three-tier dump capacity, $\mathrm{m}^{3} ; A$ - the stope width on the face side, $\mathrm{m} ; K_{p}$ - the coefficient of rock loosening in the dump.

For more detailed analysis of the internal multi-tier dumps' capacity their classification on the following criteria was made: the number of tiers (starting with two) and piling scheme of dump tiers (with the preparation of additional capacity for a particular tier or without preparation).

For the four-tier dump it is necessary to take into account the possibility of preparing the additional capacity in the second, third or in each tier.

In the first tier there is no need on preparing capacity, since the rock at the bottom of the first tier is thickened due to its stop by an explosive move. This thickening of the prism zone contributes to the dump stability.

\section{Results and Discussion}

Internal four-tier dumps, being piled in the development of three flat seams strata can be classified by four types:

Type 1 - the capacity of dump tiers is formed during working the regular stope at the face side.

Type 2 - the capacity of dump tiers is formed during working the regular stope at the face side with the preparation of additional capacity on the second tier (Fig. 1).

Type 3 - the capacity of dump tiers is formed during working the regular stope at the face side with the preparation of additional capacity on the third tier.

Type 4 - the capacity of dump tiers is formed during working the regular stope at the face side with the preparation of additional capacity on the second and third tiers.

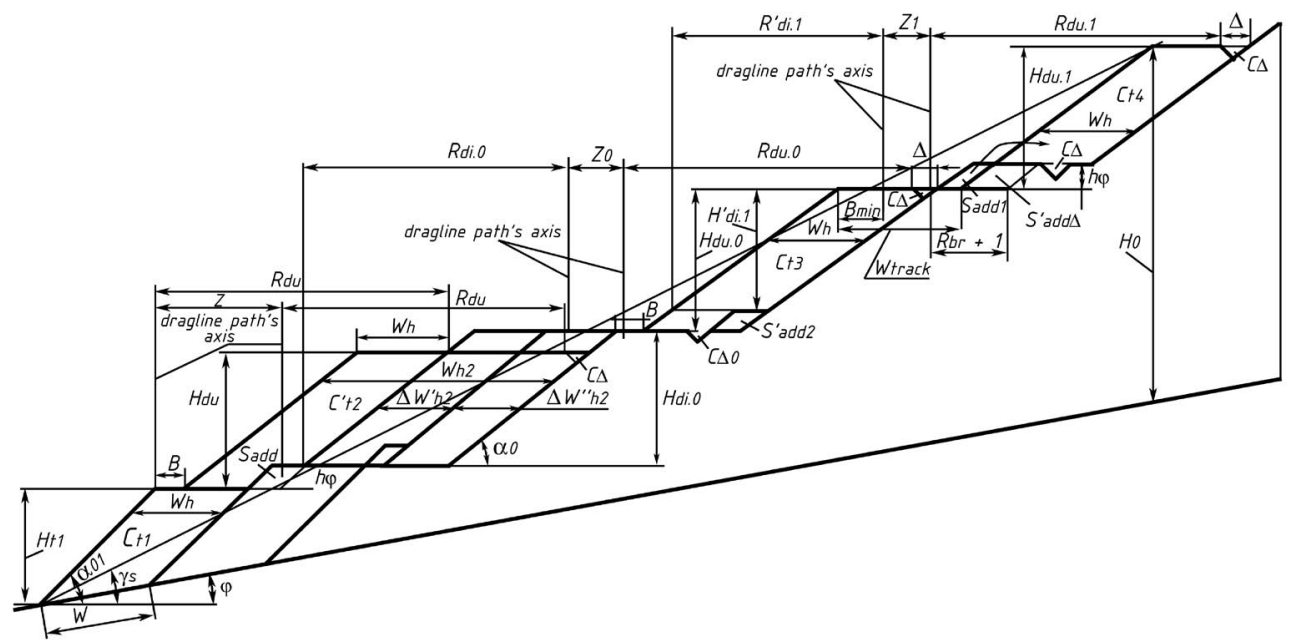

Fig. 1. The scheme of work on the dump side with the preparation of additional capacity on the second dump tier.

The schemes of dumping, being different in the preparation of additional capacity for any dump tier, have smaller capacity than schemes without capacity preparation. This provision is explained by the following. 
The width of drilling and blasting stopes, the minimum value of which is determined by the condition of the turn of the coal carriers in mining faces $(35-40 \mathrm{~m})$, does not allow fully using the radius of loading of the currently used draglines when piling up the dumping layers [5]. Thus, Fig. 2 shows the scheme of tier filling for increasing the stacking capacity of which the additional capacity is prepared.

When preparing the capacity from a permanent dump array a cutting layer of width $l_{\text {cut }}$ is taken out.

The unloading radius of used draglines (with beam length of $85-90 \mathrm{~m}$ ) makes it possible to pile the tier with the width of its upper platform along the line 6-7. When the dump is being formed at the angle of the natural slope grade $\alpha_{0}\left(\alpha_{0}=37^{0}\right)$, the prepared capacity is not completely filled (Figures 8-9-3-4 in Fig. 2). When using, for example, long-beam draglines, the rock unloading could be made in 7', which essentially increases the volume of rock placed in the prepared capacity (Figures $8^{\prime}-7^{\prime}-3-4$ in Fig. 2). With the existing parameters of used draglines (ESH 15.90, ESH 20.90, that is, the beam length is $90 \mathrm{~m}$ ), it is possible to fill the capacity ("container") (Figures 7-7-9 in Fig. 2) only in the following way: to raise the dumping dragline to the working platform of the tier (line 6-7 in Fig. 2) and to carry out an additional working stroke of the excavator. Cutting by this stroke the tier slope, it is possible to pile "the container" [6].

This method is used in the development of dumping schemes of types 2 and 4 for the piling of the second tier, since the main volumes of the rock entering the dump are accounted for it.

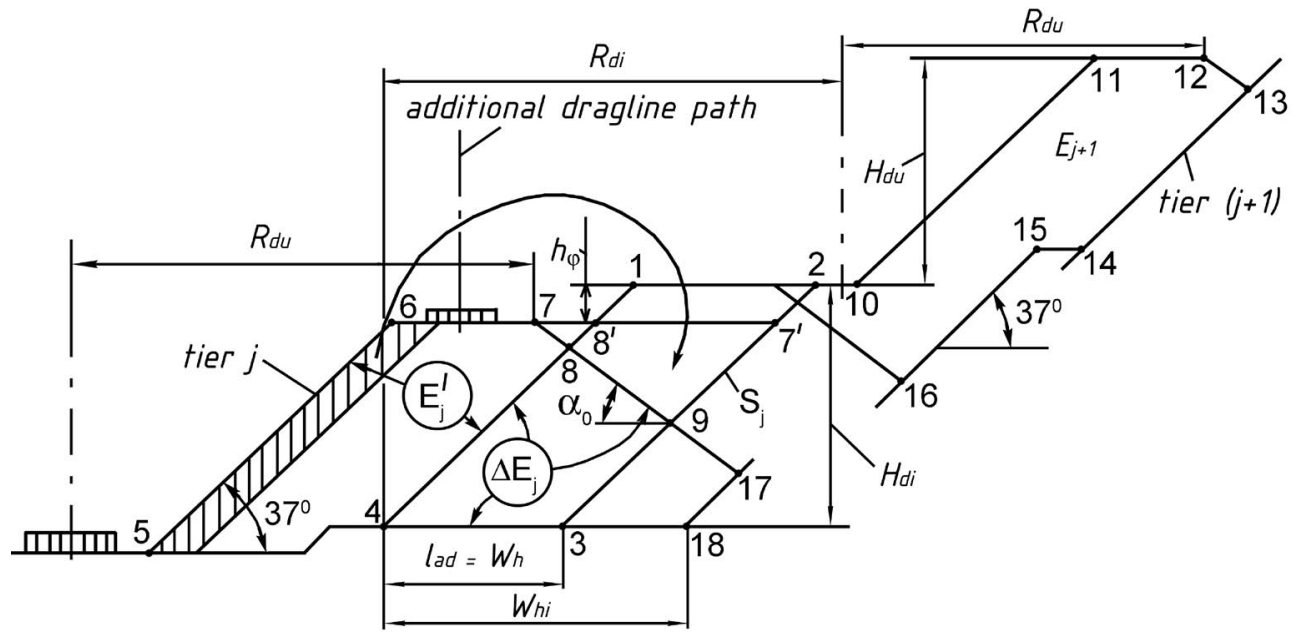

Fig. 2. The scheme of filling the tier with the preparation of additional capacity for it.

Consequently, in dumping schemes without capacity preparation overlying tier capacity $(j+1)$ is used entirely for the rock from the face side. In the scheme with capacity preparation for the incoming rock only a part of the prepared capacity is used $\Delta E_{j}<E(j+1)$.

In connection with this, the volume of the rock placed in the dumps with prepared capacity is smaller than in dumps without capacity preparation.

In case of equal withdrawal volume in the capacity preparation $S_{j}$ (aeas 2-10-11-12-1314-15-16-7' in Fig. 2), i.e. $S_{j}=E(j+1)$ and the cutting width $l_{c u t}=A_{t}$ the maximum increase in the volume of the rock, located in the prepared capacity $\Delta E j=\max$, is achieved.

To manage the volume $\Delta E j$ is possible only towards its reduction, for which $l_{\text {cut }}$ or digging depth $H_{v}$ should be reduced (Fig. 2). Controlling the volume $\Delta E j$ towards its reduction is used in this study in the development of the structures and parameters of excavation schemes. 
The volume value $\Delta E j$ is affected by the dip angle, with increasing of which parameter $A$ decreases and parameter $h_{\varphi}$ increases (Fig. 2). Their combined effect reduces the capacity of the overlying tier $E(j+1)$ and, consequently, the value $S_{j}$ and $\Delta E j$.

For quantitative estimation of the effectiveness of additional capacity preparation it is offered to estimate $K_{i . \Delta E}$ - utilization coefficient of prepared capacity, equal to the ratio of the rock volume, located in the capacity, to the total volume of worked out rock while creating the capacity.

As the graphics of piling scheme, built for draglines with various beam length, are geometrically similar figures (parameter " $\Delta$ ", combining unloading radius and oval dump contour depend only on the width of the stope), the value of the coefficient $K_{i . \Delta E}$ has a similar value for all models of draglines.

As an example, Tab. 1 shows the values $K_{i . \Delta E}$ for draglines ESH 20.90, ESH 20.100, ESH 30.110, ESH 25.120 calculated for the piling scheme of type 2 with a dip angle of $10^{\circ}$.

Table 1. $K_{i . \Delta E}$ values for various models of draglines

\begin{tabular}{|c|c|c|c|c|}
\hline \multirow{2}{*}{$\begin{array}{c}\text { Stope } \\
\text { width } \\
(\boldsymbol{A}), \mathbf{m}\end{array}$} & \multicolumn{4}{|c|}{$\boldsymbol{K}_{\boldsymbol{i} . \Delta E}$ value for draglines } \\
\cline { 2 - 5 } & ESH 20.90 & ESH 20.100 & ESH 30.110 & ESH 25.120 \\
\hline 40 & $0.826( \pm 0 \%)$ & $0.831(+0,5 \%)$ & $0.817(-1,09 \%)$ & $0.838(+1,45 \%)$ \\
\hline 45 & $0.808( \pm 0 \%)$ & $0.821(+1,6 \%)$ & $0.804(-0,4 \%)$ & $0.798(-1,02 \%)$ \\
\hline 50 & $0.786( \pm 0 \%)$ & $0.801(+1,5 \%)$ & $0.797(+1 \%)$ & $0.802(+1,3 \%)$ \\
\hline
\end{tabular}

As it can be seen from Tab. 1 when calculating $K_{i . \Delta E}$ for draglines of different models, the deviation of the coefficients $K_{i . \Delta E}$ does not exceed $\pm 1.5 \%$, which provides sufficient accuracy and reliability of calculations by the average value of this coefficient.

The average value of $K_{i . \Delta E}$ and the calculation formulas for the four-tier dumps are given in Tab. 2, and the graphs of its dependence on angle $\varphi$ and stope width $A$ are in Fig. 3-a, b.
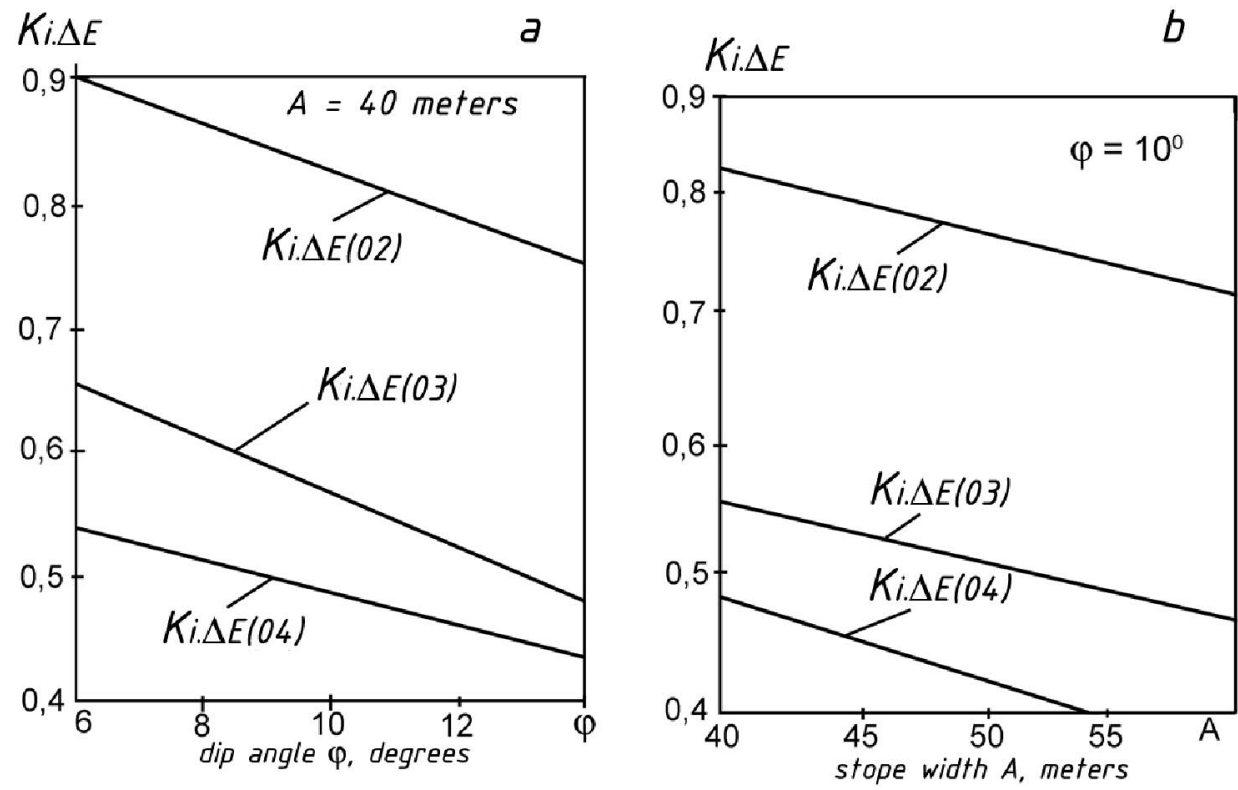

Fig. 3. The dependence of using coefficient of the prepared capacity on the basic factors: a - bedding angle of the seam; $b$ - stope width. 


\section{Conclusions}

As it is seen from the graphs, $K_{i . \Delta E}$ coefficient decreases both with increasing the bedding angle of seams and increasing the stope width.

The factor causing the decrease of $K_{i . \Delta E}$ in increasing the bedding angle of seams is the increase of parameter $h_{\varphi}$ and therefore reduction of digging possibility of a dragline $\left(H_{v}-\right.$ $\left.h_{\varphi}\right)$. As a result, the amount of additional prepared capacity reduces.

The factor causing the decrease of $K_{i . \Delta E}$ in increasing the width of the stope is a disproportionate decrease in the volume of rock coming into the prepared capacity, relatively to the excavated volume in the prepared capacity.

\section{References}

1. I. Vukotic, V. Kecojevic, W. Zhang, Q. Cai, Int. J. Min. Sci. Tech., 23(6), 901 (2013)

2. M.M. Bereznyak, A.V. Kalinin and V.G. Pronoza, Soviet Mining 6(6), 638 (1970)

3. B. Lokhanov, Y.A. Zakharov, M.M. Bereznyak, A.V. Kalinin, Sov. Min., 3(5), 523 (1967)

4. A.S. Tanaino, V.I. Cheskidov, J. Min. Sci., 35(3), 304 (1999)

5. N. Demirel, J. Min. Sci., 47(4), 441 (2011)

6. I.V. Nazarov, J. Min. Sci., 48(1), 55 (2011) 